\title{
On monotonic functions from the unit interval into a Banach space with uncountable sets of points of discontinuity
}

\author{
by
}

Artur Michalak (Poznań)

\begin{abstract}
We say that a function $f$ from $[0,1]$ to a Banach space $X$ is increasing with respect to $E \subset X^{*}$ if $x^{*} \circ f$ is increasing for every $x^{*} \in E$. We show that if $f:[0,1] \rightarrow X$ is an increasing function with respect to a norming subset $E$ of $X^{*}$ with uncountably many points of discontinuity and $Q$ is a countable dense subset of $[0,1]$, then $(1) \overline{\operatorname{lin}\{f([0,1])\}}$ contains an order isomorphic copy of $D(0,1),(2) \varlimsup \overline{\operatorname{lin}\{f(Q)\}}$ contains an isomorphic copy of $C([0,1]),(3) \overline{\operatorname{lin}\{f([0,1])\}} / \overline{\operatorname{lin}\{f(Q)\}}$ contains an isomorphic copy of $c_{0}(\Gamma)$ for some uncountable set $\Gamma$, (4) if $I$ is an isomorphic embedding of $\overline{\operatorname{lin}\{f([0,1])\}}$ into a Banach space $Z$, then no separable complemented subspace of $Z$ contains $I(\overline{\operatorname{lin}\{f(Q)\}})$.
\end{abstract}

Throughout the paper, $X$ will be a real or complex Banach space and $X^{*}$ its topological dual. We say that a function $f:[0,1] \rightarrow X$ is increasing with respect to a subset $E$ of $X^{*}$ if $x^{*} \circ f$ is increasing (= nondecreasing) for every $x^{*} \in E$. Throughout, we will assume that $E$ is a norming subset of $X^{*}$ (i.e. there exist constants $C \geq c>0$ such that $c\|x\| \leq \sup \left\{\left|x^{*}(x)\right|\right.$ : $\left.x^{*} \in E\right\} \leq C\|x\|$ for every $\left.x \in X\right)$. Every increasing function from the unit interval into a Banach lattice $F$ is increasing with respect to the positive part of the unit ball of $F^{*}$. Consequently, the definition covers all natural examples of increasing functions. On the other hand, we can consider an increasing function with respect to $E$ as an increasing function taking values in $C\left(\bar{E}^{w^{*}}\right)$. We study the properties of monotonic functions $f:[0,1] \rightarrow X$ with uncountable sets of points of discontinuity.

Real monotonic functions on $[0,1]$ have countable sets of points of discontinuity. However, not all monotonic functions from $[0,1]$ into Banach lattices have this property (see [7], [3]). A Banach lattice $F$ is said to have property $(\lambda)$ if every increasing function from $[0,1]$ into $F$ has only countably

2000 Mathematics Subject Classification: Primary 46B20.

Key words and phrases: monotonic functions in Banach spaces.

The research was supported by Komitet Badań Naukowych (State Committee for Scientific Research), Poland, grant no. 2 P03A 05115. 
many points of discontinuity. The study of properties of Banach lattices with property $(\lambda)$ was initiated by B. Lavrič in [7]. He showed that a $\sigma$-Dedekind complete Banach lattice has property $(\lambda)$ if and only if it does not contain any lattice copy of $l^{\infty}$. L. Drewnowski [3] observed that the space $D(0,1)$ of all scalar left continuous functions on $(0,1]$ with right limits on $[0,1)$, equipped with the supremum norm, contains no isomorphic copy of $l^{\infty}$ and the function $f:[0,1] \rightarrow D(0,1)$ given by $f(\alpha)=\chi_{[0, \alpha]}$ is discontinuous at every point. This shows that the space $l^{\infty}$ does not play a crucial role in the theory of monotonic functions on $[0,1]$ taking values in Banach lattices with uncountable sets of points of discontinuity, and simultaneously directs our attention to the space $D(0,1)$. We show that property $(\lambda)$ and the fact that a Banach lattice does not contain any order copy of $D(0,1)$ are equivalent (see Theorem 4(c)).

Preliminaries. We denote by $C(K)$ the Banach space of all scalar continuous functions on a compact Hausdorff space $K$ endowed with the supremum norm. For a given set $\Gamma$ the Banach space of all functions $x: \Gamma \rightarrow \mathbb{C}$ such that the set $\{\gamma \in \Gamma:|x(\gamma)|>\varepsilon\}$ is finite for every $\varepsilon>0$ equipped with the supremum norm will be denoted by $c_{0}(\Gamma)$. Moreover, the unit ball of a Banach space $X$ will be denoted by $B_{X}$ and the weak* topology of $X^{*}$ by $w^{*}$. For other notations and terminology the reader is referred to [12] and [5].

The Helly space $\mathbb{H}$ consists of all nondecreasing functions $x:[0,1] \rightarrow$ $[0,1]$ and is equipped with the pointwise convergence topology. It is compact, Hausdorff, separable and first countable (see [6, p. 164]). For $\alpha \in[0,1]$ we denote by $\pi_{\alpha}: \mathbb{H} \rightarrow[0,1]$ the function given by $\pi_{\alpha}(x)=x(\alpha)$. As observed by Drewnowski, these natural projections lead to an increasing function.

Example. Let $H$ be a closed subset of the Helly space. The function $f:[0,1] \rightarrow C(H)$ given by

$$
f(\alpha)=\pi_{\alpha}
$$

is increasing.

A fundamental role in our considerations is played by the next theorem. It shows that every increasing function in our sense generates a closed subset of the Helly space, and the properties of the function are determined by the set. More general results on relationships between increasing functions taking values in general order metric spaces and the topology of Helly-like spaces can be found in [4].

ThEOREM 1. Let $f:[0,1] \rightarrow X$ be an increasing function with respect to a norming subset $E$ of $B_{X^{*}}$ such that $f(0)=0$ and $\|f(1)\|=1$. Let $H_{f}=\left\{x^{*} \circ f: x^{*} \in \bar{E}^{w^{*}}\right\}$ be equipped with the pointwise convergence topology. Then 
(1) $H_{f}$ is a closed subset of the Helly space,

(2) if $Y$ is the closed linear hull in $C\left(H_{f}\right)$ of the functions $\pi_{\alpha}$ for $\alpha \in$ $[0,1]$, then there exists a positive isomorphic embedding $I: Y \rightarrow X$ such that $f(\alpha)=I\left(\pi_{\alpha}\right)$ for every $\alpha \in[0,1]$, where the order in $X$ is generated by $E$,

(3) moreover, the following assertions are equivalent:

(a) the function $f$ has countably many points of discontinuity,

(b) the space $H_{f}$ is metrizable,

(c) the function $f$ has separable range.

Proof. Let $\Phi_{f}:\left(\bar{E}^{w^{*}}, w^{*}\right) \rightarrow \mathbb{H}$ be given by $\Phi_{f}\left(x^{*}\right)=x^{*} \circ f$. It is clear that $\Phi_{f}$ is continuous. Thus $H_{f}$ is a closed subset of the Helly space. Since $E$ is norming, the operator $S: X \rightarrow C\left(\left(\bar{E}^{w^{*}}, w^{*}\right)\right)$ given by $S(x)=\left.x\right|_{\bar{E}^{w^{*}}}$ is an isomorphism. Let $R: C\left(H_{f}\right) \rightarrow C\left(\left(\bar{E}^{w^{*}}, w^{*}\right)\right)$ be the isometry given by $R(h)=h \circ \Phi_{f}$. Then $R\left(\pi_{\alpha}\right)\left(x^{*}\right)=x^{*}(f(\alpha))=S(f(\alpha))\left(x^{*}\right)$ for every $x^{*} \in \bar{E}^{w^{*}}$. Hence $R\left(\pi_{\alpha}\right)=S(f(\alpha))$ for every $\alpha \in[0,1]$. The operators $R$ and $S$ are isomorphic embeddings. Moreover, $x$ is a positive element of $X$ if and only if $S(x)$ is positive in $C\left(\left(\bar{E}^{w^{*}}, w^{*}\right)\right)$. Therefore the operator $I=\left.\left.S^{-1}\right|_{S(X)} \circ R\right|_{Y}$ is a positive isomorphic embedding.

(a) $\Rightarrow(\mathrm{b})$. Let $A_{f}$ be the set of all points of discontinuity of $f$. Then the set of all points of discontinuity of elements of $H_{f}$ is contained in $A_{f}$. Therefore the functions $\pi_{\alpha}$ for $\alpha \in A_{f} \cup \mathbb{Q}$ separate points of $H_{f}$. Since $H_{f}$ is Hausdorff and compact, it is metrizable.

The implication $(\mathrm{b}) \Rightarrow(\mathrm{c})$ is a straightforward consequence of $(2)$ and the fact that the space $C\left(H_{f}\right)$ is separable.

$(\mathrm{c}) \Rightarrow(\mathrm{b})$. Suppose that $f([0,1])$ is separable. Let $V$ be its closed linear hull. Then the function $\tilde{f}:[0,1] \rightarrow V$ given by $\tilde{f}(\alpha)=f(\alpha)$ for every $\alpha \in[0,1]$ is increasing with respect to the norming weak* compact subset $\left\{x^{*} \mid V: x^{*} \in \bar{E}^{w^{*}}\right\}$ of $V^{*}$. The set is metrizable in the weak ${ }^{*}$ topology of $V^{*}$ and $\Phi_{\tilde{f}}\left(\left\{x^{*} \mid V: x^{*} \in \bar{E}^{w^{*}}\right\}\right)=\Phi_{f}\left(\bar{E}^{w^{*}}\right)$. Consequently, $H_{f}$ is metrizable.

(b) $\Rightarrow(\mathrm{a})$. Let $D_{f}$ be the set of all points of discontinuity of elements of $H_{f}$. A subset of $\mathbb{H}$ is separable and metrizable (not necessarily closed) if and only if the set of all points of discontinuity of all members of the set is countable (see [8, Prop. 2]). Thus $D_{f}$ is countable. If $\beta \notin D_{f}$ and $\left(\beta_{n}\right)$ is an increasing (or decreasing) sequence in $[0,1]$ converging to $\beta$, then the sequence $\left(\pi_{\beta_{n}}\right)$ of functions converges to $\pi_{\beta}$ pointwise on $H_{f}$. Since the $\pi_{\beta_{n}}$ are continuous on $H_{f}$, the convergence is uniform by the Dini theorem. Then the function $g:[0,1] \rightarrow C\left(H_{f}\right)$ given by $g(\alpha)=\pi_{\alpha}$ is continuous on $[0,1] \backslash D_{f}$. An appeal to (2) completes the proof.

An important role in our considerations is played by the subset $\mathbb{L}=$ $\{x \in \mathbb{H}: x([0,1]) \subset\{0,1\}\}$ of $\mathbb{H}$. The space $\mathbb{L}$ is homeomorphic to the 
classical two-arrow space with two isolated points added. Consequently, it is Hausdorff, compact, sequentially compact, hereditarily separable and hereditarily Lindelöf (see [5, p. 270]). Note also that $C(\mathbb{L})$ is order isomorphic to $D(0,1)$.

FACT 2. If $H$ is a closed nonmetrizable subset of $\mathbb{H}$, then there exists a separable subset of $H$ with nonmetrizable closure.

Proof. Let $Q$ be a countable dense subset of $[0,1]$. Let $\Gamma_{1}$ be the set of all $\gamma \in[0,1] \backslash Q$ such that there exist $x_{0}, x_{1} \in H$ with $x_{0}(\alpha)=x_{1}(\alpha)$ for every $\alpha \in Q$ and $x_{0}(\gamma) \neq x_{1}(\gamma)$. The family of functions $\pi_{\alpha}$ for $\alpha \in Q \cup \Gamma_{1}$ separates points of $H$. Since $H$ is nonmetrizable, the set $\Gamma_{1}$ is uncountable. Then there exist $0 \leq a<b \leq 1$ and an uncountable subset $\Gamma_{2}$ of $\Gamma_{1}$ such that for every $\gamma \in \Gamma_{2}$ there exist $x_{0}^{\gamma}, x_{1}^{\gamma} \in H$ with $x_{0}^{\gamma}(\alpha)=x_{1}^{\gamma}(\alpha)$ for every $\alpha \in Q$ and $x_{0}^{\gamma}(\gamma) \leq a<b \leq x_{1}^{\gamma}(\gamma)$. Let $\psi:[0,1] \rightarrow[0,1]$ be a continuous nondecreasing function such that $\psi([0, a])=\{0\}$ and $\psi([b, 1])=\{1\}$. Let $\Psi: H \rightarrow[0,1]^{[0,1]}$ be given by

$$
\Psi(x)=\psi \circ x .
$$

The set $\Psi(H) \cap \mathbb{L}$ is closed, uncountable (consequently, nonmetrizable) and separable. A standard argument completes the proof.

In what follows we will also need the following well known result (a dual result for $L^{1}$ is well known; see [2, p. 211]).

FACT 3. Let $\left(e_{n}\right)$ be a sequence in a Banach space $X$ such that

(1) $e_{n}=e_{2 n}+e_{2 n+1}$ for every $n$,

(2) there exist constants $0<c \leq C$ such that for any scalars $a_{2^{n}}, \ldots$, $a_{2^{n+1}-1}$,

$$
c \max _{2^{n} \leq k<2^{n+1}}\left|a_{k}\right| \leq\left\|\sum_{k=2^{n}}^{2^{n+1}-1} a_{k} e_{k}\right\| \leq C \max _{2^{n} \leq k<2^{n+1}}\left|a_{k}\right| .
$$

Then $X$ has a subspace isomorphic to $C([0,1])$.

We present the proof for the convenience of the reader.

Proof. Let $\lambda$ be the Haar measure on $A_{1}=\{-1,1\}^{\mathbb{N}}$. For every $n \in \mathbb{N}$ and $0 \leq k \leq 2^{n}-1$ let

$$
\begin{aligned}
& A_{2^{n}+k}=\left\{\left(j_{n}\right) \in\{-1,1\}^{\mathbb{N}}: j_{l}=i_{l} \text { for } l=1, \ldots, n\right. \\
& \text { where } \left.\sum_{l=1}^{n} 2^{n-l-1}\left(i_{l}+1\right)=k\right\} .
\end{aligned}
$$

The sets $A_{2^{n}}, \ldots, A_{2^{n+1}-1}$ are pairwise disjoint, clopen in $\{-1,1\}^{\mathbb{N}}$ and $A_{2 n} \cup A_{2 n+1}=A_{n}$ for every $n \in \mathbb{N}$. It is clear that $\chi_{A_{n}}$ belongs to $C\left(\{-1,1\}^{\mathbb{N}}\right)$ 
and for every $a_{2^{n}}, \ldots, a_{2^{n+1}-1} \in \mathbb{C}$,

$$
\left\|\sum_{k=2^{n}}^{2^{n+1}-1} a_{k} \chi_{A_{k}}\right\|=\max _{2^{n} \leq k<2^{n+1}-1}\left|a_{k}\right| \text {. }
$$

Let $T_{n}: C\left(\{-1,1\}^{\mathbb{N}}\right) \rightarrow X$ and $S_{n}: C\left(\{-1,1\}^{\mathbb{N}}\right) \rightarrow C\left(\{-1,1\}^{\mathbb{N}}\right)$ be the linear operators given by

$$
T_{n}(f)=\sum_{k=2^{n}}^{2^{n+1}-1} e_{k} \frac{1}{\lambda\left(A_{k}\right)} \int_{A_{k}} f d \lambda, \quad S_{n}(f)=\sum_{k=2^{n}}^{2^{n+1}-1} \chi_{A_{k}} \frac{1}{\lambda\left(A_{k}\right)} \int_{A_{k}} f d \lambda .
$$

It is clear that $c\left\|S_{n}(f)\right\| \leq\left\|T_{n}(f)\right\| \leq C\left\|S_{n}(f)\right\|$ and $c\left\|S_{n+k}(f)-S_{n}(f)\right\| \leq$ $\left\|T_{n+k}(f)-T_{n}(f)\right\| \leq C\left\|S_{n+k}(f)-S_{n}(f)\right\|$ for all $n, k \in \mathbb{N}$. Since $f=$ $\lim _{n} S_{n}(f)$ for every $f \in C\left(\{-1,1\}^{\mathbb{N}}\right)$, the operator $T: C\left(\{-1,1\}^{\mathbb{N}}\right) \rightarrow X$ given by $T(f)=\lim _{n} T_{n}(f)$ is an isomorphism. An appeal to the Milyutin theorem completes the proof.

Main results. For $\gamma \in[0,1]$ and $s \in\{0,1\}$ we denote by $y_{s}^{\gamma}$ the following element of $\mathbb{H}$ :

$$
y_{s}^{\gamma}(\alpha)= \begin{cases}0 & \text { if } \alpha<\gamma \\ s & \text { if } \alpha=\gamma \\ 1 & \text { if } \alpha>\gamma\end{cases}
$$

For a closed subset $\Gamma$ of $[0,1]$ we put $\mathbb{L}_{\Gamma}=\left\{y_{s}^{\gamma}: \gamma \in \Gamma, s \in\{0,1\}\right\}$. Then $\mathbb{L}=\mathbb{L}_{[0,1]}$. It is easy to see that the map $m: \mathbb{L} \rightarrow[0,1]$ given by $m\left(y_{s}^{\gamma}\right)=\gamma$ is continuous. Consequently, $\mathbb{L}_{\Gamma}$ is a compact subset of $\mathbb{L}$ for every closed subset $\Gamma$ of $[0,1]$. For any $\gamma_{1}<\ldots<\gamma_{n}$ in $\Gamma$ and $g_{1}, \ldots, g_{n}, g_{n+1} \in \mathbb{C}$ we have

$$
\begin{aligned}
& \left\|g_{n+1}+\sum_{i=1}^{n} g_{i} \pi_{\gamma_{i}}\right\|_{C\left(\mathbb{L}_{\Gamma}\right)} \\
& =\left\|\left(\sum_{i=1}^{n+1} g_{i}\right) \pi_{\gamma_{1}}+\sum_{i=2}^{n}\left(\sum_{j=i}^{n+1} g_{j}\right)\left(\pi_{\gamma_{i}}-\pi_{\gamma_{i-1}}\right)+g_{n+1}\left(1-\pi_{\gamma_{n}}\right)\right\|_{C\left(\mathbb{L}_{\Gamma}\right)} \\
& =\max \left\{\left|\sum_{j=i}^{n+1} g_{j}\right|: i=1, \ldots, n+1\right\} .
\end{aligned}
$$

Moreover, the function $g_{n+1}+\sum_{i=1}^{n} g_{i} \pi_{\gamma_{i}}$ is nonnegative on $\mathbb{L}_{\Gamma}$ if and only if $\min \left\{\sum_{i=j}^{n+1} g_{i}: j=1, \ldots, n+1\right\} \geq 0$. Applying the fact that on $\mathbb{L}$,

$$
\pi_{\gamma_{1}} \pi_{\gamma_{2}}= \begin{cases}\pi_{\gamma_{1}} & \text { if } \gamma_{1} \leq \gamma_{2} \\ \pi_{\gamma_{2}} & \text { if } \gamma_{1} \geq \gamma_{2}\end{cases}
$$

and the Stone-Weierstrass theorem, we find that the closed linear hull of 
$\left\{\pi_{\gamma}: \gamma \in \Gamma\right\} \cup\{1\}$ in $C\left(\mathbb{L}_{\Gamma}\right)$ coincides with $C\left(\mathbb{L}_{\Gamma}\right)$. Therefore, if $\varphi: \Gamma_{1} \rightarrow \Gamma_{2}$ is an increasing function, then the operator $M_{\varphi}: \mathbb{L}_{\Gamma_{1}} \rightarrow \mathbb{L}_{\Gamma_{2}}$ given by $M_{\varphi}\left(\pi_{\gamma}\right)=\pi_{\varphi(\gamma)}$ has a continuous extension. If additionally $\varphi$ is surjective, then selecting exactly one element $\psi(\gamma)$ in $\varphi^{-1}(\gamma)$ we see that the subspace $\overline{\operatorname{lin}\left\{\pi_{\psi(\gamma)}: \gamma \in \Gamma_{2}\right\}}$ of $C\left(\mathbb{L}_{\Gamma_{1}}\right)$ is order isometric to $C\left(\mathbb{L}_{\Gamma_{2}}\right)$. The fundamental properties of monotonic functions $f:[0,1] \rightarrow X$ are gathered in the theorem below. Part (b) of the theorem is closely related to H. Corson's result that the quotient space $D(0,1) / C([0,1])$ is isomorphic to $c_{0}([0,1])$ (see $[1]$ ).

TheOREM 4. If there exists an increasing function $f:[0,1] \rightarrow X$ with respect to a norming subset $E$ of $X^{*}$ with uncountably many points of discontinuity, then

(a) the closed linear hull of $f(Q)$ contains a subspace isomorphic to $C([0,1])$ for every countable dense subset $Q$ of $[0,1]$,

(b) for every countable dense subset $Q$ of $[0,1]$ the quotient space $X / \overline{\operatorname{lin} f(Q)}$ has a subspace isomorphic to $c_{0}(\Gamma)$ where $\Gamma$ is an uncountable set,

(c) there exists a positive isomorphic embedding of $C(\mathbb{L})$ into the closed linear hull of $f([0,1])$.

Proof. We can assume that $f(0)=0,\|f(1)\|=1$ and $E \subset B_{X^{*}}$. Let $H_{f}$ be the closed nonmetrizable subset of $\mathbb{H}$ associated with $f$ as in Theorem 1 . Let $Q$ be a countable dense subset of $[0,1]$. Let $\Gamma_{1}$ be the set of all $\gamma \in$ $[0,1] \backslash Q$ such that there exist $x_{0}, x_{1} \in H_{f}$ with $x_{0}(\alpha)=x_{1}(\alpha)$ for every $\alpha \in Q$ and $x_{0}(\gamma) \neq x_{1}(\gamma)$. The family $\left\{\pi_{\alpha}: \alpha \in Q \cup \Gamma_{1}\right\}$ separates points of $H_{f}$. Since $H_{f}$ is nonmetrizable, the set $\Gamma_{1}$ is uncountable. Thus there exist $0 \leq a<b \leq 1$ and an uncountable subset $\Gamma_{2}$ of $\Gamma_{1}$ such that for every $\gamma \in \Gamma_{2}$ there exist $x_{0}^{\gamma}, x_{1}^{\gamma} \in H$ with $x_{0}^{\gamma}(\alpha)=x_{1}^{\gamma}(\alpha)$ for every $\alpha \in Q$ and $x_{0}^{\gamma}(\gamma) \leq a<b \leq x_{1}^{\gamma}(\gamma)$. For every $\gamma \in \Gamma_{2}$ there exists $\delta_{\gamma}>0$ such that

$$
x_{0}^{\gamma}\left(\gamma+\delta_{\gamma}\right)-\lim _{\alpha \rightarrow \gamma+} x_{0}^{\gamma}(\alpha)+\lim _{\alpha \rightarrow \gamma-} x_{0}^{\gamma}(\alpha)-x_{0}^{\gamma}\left(\gamma-\delta_{\gamma}\right) \leq C,
$$

where $C=(b-a) / 2$ in the proof of (a) and (b), and $C=(b-a)^{2} / 8$ in the proof of (c). It is clear that there exists an uncountable subset $\Gamma_{3}$ of $\Gamma_{2}$ and $\delta>0$ such that $\delta_{\gamma}>\delta$ for every $\gamma \in \Gamma_{3}$. Since $[0,1]$ can be covered by finitely many intervals of length $\delta$, there exist $0 \leq c<d \leq 1$ with $d-c<\delta$ such that the set $\Gamma_{4}=\Gamma_{3} \cap(c, d)$ is uncountable. At this point we divide the proof into three parts.

(a) Let $P=\left\{k / 2^{n}: n \in \mathbb{N}, k=0, \ldots, 2^{n}\right\}$. Since $\Gamma_{4}$ is uncountable, there exists a strictly increasing function $\sigma: P \rightarrow Q \cap(c, d)$ such that the interval $(\sigma(s), \sigma(t))$ contains uncountably many elements of $\Gamma_{4}$ for every $s, t \in P$ with $s<t$. Let

$$
e_{2^{n}+k}=\pi_{\sigma\left((k+1) / 2^{n}\right)}-\pi_{\sigma\left(k / 2^{n}\right)}
$$


and let $\omega(n, k)$ be a member of $\Gamma_{4} \cap\left(\sigma\left(k / 2^{n}\right), \sigma\left((k+1) / 2^{n}\right)\right)$ for every $n \in \mathbb{N}$ and $k=0, \ldots, 2^{n}-1$. Then for $g_{2^{n}}, \ldots, g_{2^{n+1}-1} \in \mathbb{C}$ and $j=0, \ldots, 2^{n}-1$,

$$
\begin{aligned}
& \left\|\sum_{k=2^{n}}^{2^{n+1}-1} g_{k} e_{k}\right\| \geq\left|\sum_{k=0}^{2^{n}-1} g_{2^{n}+k}\left(\pi_{\sigma\left((k+1) / 2^{n}\right)}-\pi_{\sigma\left(k / 2^{n}\right)}\right)\left(x_{0}^{\omega(n, j)}\right)\right| \\
& \geq(b-a)\left|g_{2^{n}+j}\right| \\
& -\max _{0 \leq k<2^{n}}\left|g_{2^{n}+k}\right| \sum_{k \neq j}\left(x_{0}^{\omega(n, j)}\left(\sigma\left(\frac{k+1}{2^{n}}\right)\right)-x_{0}^{\omega(n, j)}\left(\sigma\left(\frac{k}{2^{n}}\right)\right)\right) \\
& \geq(b-a)\left|g_{2^{n}+j}\right|-\max _{0 \leq k<2^{n}}\left|g_{2^{n}+k}\right|\left(x_{0}^{\gamma}(d)-\lim _{\alpha \rightarrow \omega(n, j)+} x_{0}^{\omega(n, j)}(\alpha)\right. \\
& \left.+\lim _{\alpha \rightarrow \omega(n, j)-} x_{0}^{\omega(n, j)}(\alpha)-x_{0}^{\gamma}(c)\right) \\
& \geq(b-a)\left(\left|g_{2^{n}+j}\right|-\frac{1}{2} \max _{0 \leq k<2^{n}}\left|g_{2^{n}+k}\right|\right) \text {. }
\end{aligned}
$$

Hence

$$
\left\|\sum_{k=2^{n}}^{2^{n+1}-1} g_{k} e_{k}\right\| \geq \frac{b-a}{2} \max _{2^{n} \leq k<2^{n+1}}\left|g_{k}\right|
$$

It is clear that

$$
\left\|\sum_{k=2^{n}}^{2^{n+1}-1} g_{k} e_{k}\right\| \leq \max _{2^{n} \leq k<2^{n+1}}\left|g_{k}\right| .
$$

In view of Fact 3 and Theorem 1 the closed linear hull of $f(Q)$ contains a copy of $C([0,1])$.

(b) Let $U$ be the closed linear hull of $\left\{\pi_{\gamma}: \gamma \in Q\right\}$ in $C\left(H_{f}\right)$. Denote by $L: C\left(H_{f}\right) \rightarrow C\left(H_{f}\right) / U$ the quotient map. We show that the closed linear hull of $L\left(\pi_{\alpha}\right)$ for $\alpha \in \Gamma_{4}$ is isomorphic to $c_{0}\left(\Gamma_{4}\right)$. Let $\gamma_{1}, \ldots, \gamma_{n} \in \Gamma_{4}$ and $g_{1}, \ldots, g_{n} \in \mathbb{C}$. We can assume that $0<\gamma_{1}<\ldots<\gamma_{n}$. Applying the fact that $2 \max \{|r+s|,|r+t|\} \geq|s-t|$ for any $r, s, t \in \mathbb{C}$, we get, for all $\alpha_{1}, \ldots, \alpha_{k} \in Q, h_{1}, \ldots, h_{k} \in \mathbb{C}$ and $j=1, \ldots, n$,

$$
\begin{aligned}
& \max _{s \in\{0,1\}}\left|\left(\sum_{i=1}^{n} g_{i} \pi_{\gamma_{i}}-\sum_{i=1}^{k} h_{i} \pi_{\alpha_{i}}\right)\left(x_{s}^{\gamma_{j}}\right)\right| \geq \max _{s \in\{0,1\}}\left|\sum_{i=1}^{n} g_{i} x_{s}^{\gamma_{j}}\left(\gamma_{i}\right)-\sum_{i=1}^{k} a_{i} x_{1}^{\gamma_{j}}\left(\alpha_{i}\right)\right| \\
& \geq \frac{1}{2}\left|g_{j}\left(x_{1}^{\gamma_{j}}\left(\gamma_{j}\right)-x_{0}^{\gamma_{j}}\left(\gamma_{j}\right)\right)+\sum_{i \neq j} g_{i}\left(x_{1}^{\gamma_{j}}\left(\gamma_{i}\right)-x_{0}^{\gamma_{j}}\left(\gamma_{i}\right)\right)\right| \\
& \geq \frac{1}{2}\left((b-a)\left|g_{j}\right|-\max _{i \neq j}\left|g_{i}\right|\left(x_{1}^{\gamma_{j}}(d)-\lim _{\tau \rightarrow \gamma_{j}+} x_{1}^{\gamma_{j}}(\tau)-\lim _{\tau \rightarrow \gamma_{j}-} x_{1}^{\gamma_{j}}(\tau)-x_{1}^{\gamma_{j}}(c)\right)\right) \\
& \geq \frac{b-a}{2}\left(\left|g_{j}\right|-\frac{1}{2} \max _{i \neq j}\left|g_{i}\right|\right) .
\end{aligned}
$$


Hence

$$
\left\|L\left(\sum_{i=1}^{n} g_{i} \pi_{\gamma_{i}}\right)\right\| \geq \frac{b-a}{4} \max _{1 \leq i \leq n}\left|g_{i}\right| .
$$

On the other hand, if $\beta_{i} \in Q$ and $\beta_{i}<\gamma_{i}<\beta_{i+1}$ for $i=1, \ldots, n-1$, then

$$
\begin{aligned}
\left\|L\left(\sum_{i=1}^{n} g_{i} \pi_{\gamma_{i}}\right)\right\| & \leq \max _{x \in H}\left\|\left(\sum_{i=1}^{n} g_{i} \pi_{\gamma_{i}}-\sum_{i=1}^{n} g_{i} \pi_{\beta_{i}}\right)(x)\right\| \\
& \leq \sum_{i=1}^{n}\left|g_{i}\right|\left(x\left(\gamma_{i}\right)-x\left(\beta_{i}\right)\right) \leq \max _{1 \leq i \leq n}\left|g_{i}\right| \sum_{i=1}^{n}\left(x\left(\gamma_{i}\right)-x\left(\beta_{i}\right)\right) \\
& \leq \max _{1 \leq i \leq n}\left|g_{i}\right| .
\end{aligned}
$$

Thus we show that the vectors $L\left(\pi_{\gamma}\right)$ for $\gamma \in \Gamma_{4}$ span a copy of $c_{0}\left(\Gamma_{4}\right)$ in $C\left(H_{f}\right) / U$. An appeal to Theorem 1 completes the proof of this part.

(c) Let $\Gamma_{5}$ be the closure of $\Gamma_{4}$ in $[0,1]$. Let $(0,1) \backslash \Gamma_{5}=\bigcup_{n=1}^{\infty}\left(\alpha_{n}, \beta_{n}\right)$, where distinct intervals from the sequence $\left(\left(\alpha_{n}, \beta_{n}\right)\right)$ are disjoint. For every $\gamma \in \Gamma_{5} \backslash\left(\left\{\beta_{n}: n \in \mathbb{N}\right\}\right)$ there exists an increasing sequence of elements of $\Gamma_{5}$ converging to $\gamma$ and for every $\gamma \in \Gamma_{5} \backslash\left(\left\{\alpha_{n}: n \in \mathbb{N}\right\}\right)$ there exists a decreasing sequence of elements of $\Gamma_{5}$ converging to $\gamma$. Let $z_{1}^{\gamma}$ for $\gamma \in \Gamma \backslash\left\{\beta_{n}: n \in \mathbb{N}\right\}$ be any cluster points of a sequence $\left(x_{0}^{\gamma_{n}}\right)$ where $\left(\gamma_{n}\right)$ is an increasing sequence converging to $\gamma$. The point $z_{1}^{\gamma}$ is an element of $H_{f}, \lim _{\alpha \rightarrow \gamma-} z_{1}^{\gamma}(\alpha) \leq a$, $z_{1}^{\gamma}(\gamma) \geq b$ and

$$
z_{1}^{\gamma}(d)-\lim _{\alpha \rightarrow \gamma+} z_{1}^{\gamma}(\alpha)+\lim _{\alpha \rightarrow \gamma-} z_{1}^{\gamma}(\alpha)-z_{1}^{\gamma}(c) \leq \frac{(b-a)^{2}}{8}=C .
$$

Let $z_{0}^{\gamma}$ for $\gamma \in \Gamma_{5} \backslash\left\{\alpha_{n}: n \in \mathbb{N}\right\}$ be any cluster point of a sequence $\left(x_{0}^{\gamma_{n}}\right)$ where $\left(\gamma_{n}\right)$ is a decreasing sequence converging to $\gamma$. The point $z_{0}^{\gamma}$ is an element of $H_{f}, z_{0}^{\gamma}(\gamma) \leq a, \lim _{\alpha \rightarrow \gamma+} z_{0}^{\gamma}(\alpha) \geq b$ and

$$
z_{1}^{\gamma}(d)-\lim _{\alpha \rightarrow \gamma+} z_{1}^{\gamma}(\alpha)+\lim _{\alpha \rightarrow \gamma-} z_{1}^{\gamma}(\alpha)-z_{1}^{\gamma}(c) \leq C .
$$

There exists a subset $\Gamma_{6}$ of $\Gamma_{5} \cap(c, d)$ homeomorphic to the Cantor set and an increasing continuous bijection $b:\{0,1\}^{\mathbb{N}} \rightarrow \Gamma_{6}$. Let $\Gamma=b\left(\left\{\left(\varepsilon_{n}\right) \in\{0,1\}^{\mathbb{N}}\right.\right.$ : $\varepsilon_{2 i-1}=\varepsilon_{2 i}$ for $\left.\left.i \in \mathbb{N}\right\}\right)$. Let $(0,1) \backslash \Gamma=\bigcup_{n=1}^{\infty}\left(\varrho_{n}, \eta_{n}\right)$, where distinct intervals from the sequence $\left(\left(\varrho_{n}, \eta_{n}\right)\right)$ are disjoint. The last step of the construction of $\Gamma$ implies that for every $n \in \mathbb{N}$ there exists $\tau_{n} \in \Gamma_{5} \cap\left(\varrho_{n}, \eta_{n}\right)$ such that $z_{0}^{\tau_{n}}$ and $z_{1}^{\tau_{n}}$ are elements of $H_{f}$. Let $\gamma_{1}, \ldots, \gamma_{n} \in \Gamma$ and $g_{1}, \ldots, g_{n}, g_{n+1} \in \mathbb{C}$. We can assume that $\gamma_{1}<\ldots<\gamma_{n}$. Then

$$
\begin{aligned}
\left\|g_{n+1}+\sum_{i=1}^{n} g_{i} \pi_{\gamma_{i}}\right\|_{C\left(H_{f}\right)} \leq\left\|g_{n+1}+\sum_{i=1}^{n} g_{i} \pi_{\gamma_{i}}\right\|_{C(\mathbb{H})} \\
=\left\|\left(\sum_{i=1}^{n+1} g_{i}\right) \pi_{\gamma_{1}}+\sum_{i=2}^{n}\left(\sum_{j=i}^{n+1} g_{j}\right)\left(\pi_{\gamma_{i}}-\pi_{\gamma_{i-1}}\right)+g_{n+1}\left(1-\pi_{\gamma_{n}}\right)\right\|_{C(\mathbb{H})}
\end{aligned}
$$




$$
=\max \left\{\left|\sum_{j=i}^{n+1} g_{j}\right|: i=1, \ldots, n+1\right\}=\left\|g_{n+1}+\sum_{i=1}^{n} g_{i} \pi_{\gamma_{i}}\right\|_{C\left(\mathbb{L}_{\Gamma}\right)} .
$$

Since $\Gamma$ is nowhere dense, there exist $\tau_{k_{1}}, \ldots, \tau_{k_{n}}$ such that $\tau_{k_{i}}<\gamma_{i}<\tau_{k_{i+1}}$ for every $i=1, \ldots, n$. Then for $l=2, \ldots, n$,

$$
\begin{aligned}
& \left\|\left|g_{n+1}+\sum_{i=1}^{n} g_{i} \pi_{\gamma_{i}} \|_{C\left(H_{f}\right)} \geq\right| g_{n+1}+\sum_{i=1}^{n} g_{i} \pi_{\gamma_{i}}\left(z_{1}^{\tau_{k_{l}}}\right) \mid\right. \\
& \geq\left|\left(\sum_{i=1}^{n+1} g_{i}\right) \pi_{\gamma_{1}}\left(z_{1}^{\tau_{k_{l}}}\right)+\sum_{i=2}^{n}\left(\sum_{j=i}^{n+1} g_{j}\right)\left(\pi_{\gamma_{i}}-\pi_{\gamma_{i-1}}\right)\left(z_{1}^{\tau_{k_{l}}}\right)+g_{n+1}\left(1-\pi_{\gamma_{n}}\right)\left(z_{1}^{\tau_{k_{l}}}\right)\right| \\
& \geq(b-a)\left|\sum_{j=l}^{n+1} g_{j}\right|-C \max \left\{\left|\sum_{j=i}^{n+1} g_{j}\right|: i \neq 1, l, n+1\right\}-a\left|\sum_{i=1}^{n+1} g_{i}\right|-(1-b)\left|g_{n+1}\right|
\end{aligned}
$$

and

$$
\begin{aligned}
\| g_{n+1}+ & \sum_{i=1}^{n} g_{i} \pi_{\gamma_{i}} \|_{C\left(H_{f}\right)} \geq\left|g_{n+1}+\sum_{i=1}^{n} g_{i} \pi_{\gamma_{i}}\left(z_{1}^{\tau_{k_{1}}}\right)\right| \\
& \geq b\left|\sum_{i=1}^{n+1} g_{i}\right|-C \max \left\{\left|\sum_{l=i}^{n+1} g_{l}\right|: i \neq 1, n+1\right\}-(1-b)\left|g_{n+1}\right|,
\end{aligned}
$$

and

$$
\begin{aligned}
\| g_{n+1}+ & \sum_{i=1}^{n} g_{i} \pi_{\gamma_{i}} \|_{C\left(H_{f}\right)} \geq\left|g_{n+1}+\sum_{i=1}^{n} g_{i} \pi_{\gamma_{i}}\left(z_{1}^{\tau_{k_{n+1}}}\right)\right| \\
& \geq(1-a)\left|g_{n+1}\right|-a\left|\sum_{i=1}^{n+1} g_{i}\right|-C \max \left\{\left|\sum_{l=i}^{n+1} g_{l}\right|: i \neq 1, n+1\right\} .
\end{aligned}
$$

If

$$
\begin{aligned}
\max \left\{4\left|\sum_{i=1}^{n+1} g_{i}\right|\left(a+\frac{b-a}{4}\right), 4\left|g_{n+1}\right|\right. & \left.\left.(1-b)+\frac{b-a}{4}\right)\right\} \\
& \leq \max \left\{\left|\sum_{l=i}^{n+1} g_{l}\right|: i \neq 1, n+1\right\}(b-a),
\end{aligned}
$$

then

$$
\begin{aligned}
\left\|g_{n+1}+\sum_{i=1}^{n} g_{i} \pi_{\gamma_{i}}\right\|_{C\left(H_{f}\right)} & \geq \max \left\{\left|\sum_{l=i}^{n+1} g_{l}\right|: i \neq 1, n+1\right\} \frac{b-a}{4} \\
& \geq \frac{b-a}{4}\left\|g_{n+1}+\sum_{i=1}^{n} g_{i} \pi_{\gamma_{i}}\right\|_{C\left(\mathbb{L}_{\Gamma}\right)} .
\end{aligned}
$$


If

$$
\begin{aligned}
\max \left\{4\left|g_{n+1}\right|\left((1-b)+\frac{b-a}{4}\right), \max \left\{\left|\sum_{l=i}^{n+1} g_{l}\right|: i \neq 1, n+1\right\}(b-a)\right\} & \leq 4\left|\sum_{i=1}^{n+1} g_{i}\right|\left(a+\frac{b-a}{4}\right)
\end{aligned}
$$

then

$$
\left\|g_{n+1}+\sum_{i=1}^{n} g_{i} \pi_{\gamma_{i}}\right\|_{C\left(H_{f}\right)} \geq\left|\sum_{i=1}^{n+1} g_{i}\right| \frac{b-a}{8} \geq \frac{(b-a)^{2}}{64}\left\|g_{n+1}+\sum_{i=1}^{n} g_{i} \pi_{\gamma_{i}}\right\|_{C\left(\mathbb{L}_{\Gamma}\right)}
$$

If

$$
\begin{aligned}
\max \left\{4\left|\sum_{i=1}^{n+1} g_{i}\right|\left(a+\frac{b-a}{4}\right), \max \left\{\left|\sum_{l=i}^{n+1} g_{l}\right|: i \neq 1, n+1\right\}(b-a)\right\} & \leq 4\left|g_{n+1}\right|\left((1-b)+\frac{b-a}{4}\right)
\end{aligned}
$$

then

$$
\left\|g_{n+1}+\sum_{i=1}^{n} g_{i} \pi_{\gamma_{i}}\right\|_{C\left(H_{f}\right)} \geq\left|g_{n+1}\right| \frac{b-a}{8} \geq \frac{(b-a)^{2}}{64}\left\|g_{n+1}+\sum_{i=1}^{n} g_{i} \pi_{\gamma_{i}}\right\|_{C\left(\mathbb{L}_{\Gamma}\right)} .
$$

Thus we have shown that the operator $I: C\left(\mathbb{L}_{\Gamma}\right) \rightarrow C\left(H_{f}\right)$ given by $I\left(\pi_{\gamma}\right)=\pi_{\gamma}$ is an isomorphic embedding. Moreover, the embedding is positive. It is clear that there exists an increasing surjection from $\Gamma$ onto $[0,1]$. Consequently, the space $C\left(\mathbb{L}_{\Gamma}\right)$ contains a positive and isometric copy of $C(\mathbb{L})$. An appeal to Theorem 1 completes the proof.

As a straightforward consequence of Theorem 4(c) we get

Corollary 5. A Banach lattice $F$ has property $(\lambda)$ if and only if it does not contain any order copy of $D(0,1)$.

Corollary 6. Let $f:[0,1] \rightarrow X$ be an increasing function with respect to a norming subset $E$ of $X^{*}$. Let $Q$ be a countable dense subset of $[0,1]$. If $f$ has uncountably many points of discontinuity and $I$ is an isomorphic embedding of $\overline{\operatorname{lin} f([0,1])}$ into a Banach space $Z$, then no separable complemented subspace of $Z$ contains $I(\overline{\operatorname{lin} f(Q)})$.

Proof. We can assume that $f(0)=0,\|f(1)\|=1$ and $E \subset B_{X^{*}}$. Let $H_{f}$ be the closed nonmetrizable subset of $\mathbb{H}$ associated with $f$ as in Theorem 1 . Let $U$ and $V$ be the closed linear hulls in $C\left(H_{f}\right)$ of $\left\{\pi_{\gamma}: \gamma \in Q\right\}$ and $\left\{\pi_{\gamma}: \gamma \in[0,1]\right\}$, respectively. Let $I: V \rightarrow Z$ be an isomorphic embedding. Suppose that a separable complemented subspace $Y$ of $Z$ contains $I(U)$. Let $W$ be the closed hull of $I(V)+Y$ in $Z$. Let $P: W \rightarrow W$ be a projection such that $P(W)=Y$ and $P(y)=y$ for every $y \in Y$. Let the operator 
$T: V / U \rightarrow$ ker $P$ be given by $T(f+U)=I(f)-P(I(f))$. The subset $I^{-1}(I(V) \cap P(I(V)))$ of $V$ is separable. Thus there exists a countable subset $Q_{1}$ of $[0,1]$ such that $I^{-1}(I(V) \cap P(I(V)))$ is contained in the closed linear hull of the functions $\pi_{\alpha}$ for $\alpha \in Q_{1}$ in $C\left(H_{f}\right)$. Let $\widetilde{U}$ be the closed linear hull of $\left\{\pi_{\alpha}: \alpha \in Q \cup Q_{1}\right\}$ in $C(H)$. Let $H_{1}$ be a closed, separable, nonmetrizable subset of $H_{f}$ (existing by Fact 2). Since $H_{1}$ is nonmetrizable, as in the proof of Theorem 4(b) we find an uncountable subset $\Gamma$ of $[0,1], \varepsilon>0$ and $0 \leq p<q \leq 1$ such that $\Gamma \subset(p, q)$ and for every $\gamma \in \Gamma$ there exist $x_{0}^{\gamma}, x_{1}^{\gamma} \in H_{1}$ with $x_{0}^{\gamma}(\alpha)=x_{1}^{\gamma}(\alpha)$ for every $\alpha \in Q \cup Q_{1}, x_{1}^{\gamma}(\gamma)-x_{0}^{\gamma}(\gamma) \geq \varepsilon$ and

$$
x_{1}^{\gamma}(q)-\lim _{\alpha \rightarrow \gamma+} x_{1}^{\gamma}(\alpha)+\lim _{\alpha \rightarrow \gamma-} x_{1}^{\gamma}(\alpha)-x_{1}^{\gamma}(p) \leq \varepsilon / 2 .
$$

Let $L: V \rightarrow V / U$ be the quotient map. Let $J: c_{0}(\Gamma) \rightarrow V / U$ be the isomorphic embedding from the proof of Theorem $4(\mathrm{~b})$, i.e. $J\left(e_{\gamma}\right)=L\left(\pi_{\gamma}\right)$ for every $\gamma \in \Gamma$ where $e_{\gamma}$ is the $\gamma$ th unit vector of $c_{0}(\Gamma)$. Since $Y$ is separable, there exist $\delta>0$ and an uncountable subset $\Gamma_{1}$ of $\Gamma$ such that $\left\|T\left(J\left(e_{\gamma}\right)\right)\right\|>\delta$ for every $\gamma \in \Gamma_{1}$. According to a result of H. P. Rosenthal (see [11, Thm. 3.4]) there exists an uncountable subset $\Gamma_{2}$ of $\Gamma_{1}$ such that $T \circ J: c_{0}\left(\Gamma_{2}\right) \rightarrow \operatorname{ker} P$ is an isomorphism. Let $S: W \rightarrow W / I(V)$ be the quotient map. It is clear that $W / I(V)$ is separable. Applying once again Rosenthal's result we find an uncountable subset $\Gamma_{3}$ of $\Gamma_{2}$ such that $S\left(T\left(J\left(c_{0}\left(\Gamma_{3}\right)\right)\right)\right)=0$. Therefore $I^{-1} \circ T \circ J: c_{0}\left(\Gamma_{3}\right) \rightarrow V$ is an isomorphic embedding. Moreover, $I^{-1}\left(T\left(L\left(\pi_{\gamma}\right)\right)\right) \in \pi_{\gamma}+\widetilde{U}$ for every $\gamma \in \Gamma_{3}$. Let $R: C\left(H_{f}\right) \rightarrow C\left(H_{1}\right)$ be given by $R(f)=\left.f\right|_{H_{1}}$. Let $V_{1}$ and $U_{1}$ be the closed linear hulls in $C\left(H_{1}\right)$ of $\left\{\pi_{\alpha}: \alpha \in[0,1]\right\}$ and $\left\{\pi_{\alpha}: \alpha \in Q \cup Q_{1}\right\}$, respectively. Let $L_{1}: V_{1} \rightarrow V_{1} / U_{1}$ be the quotient map. According to the proof of Theorem 5 the closed linear hull of $\left\{L_{1}\left(\pi_{\gamma}\right): \gamma \in \Gamma_{3}\right\}$ is isomorphic to $c_{0}\left(\Gamma_{3}\right)$. Since $R\left(\pi_{\alpha}\right)=\pi_{\alpha}$ for every $\alpha \in[0,1]$, we have $R(V) \subset V_{1}$ and $R(\widetilde{U}) \subset U_{1}$. Therefore $L_{1}\left(R\left(I^{-1}\left(T\left(L\left(\pi_{\gamma}\right)\right)\right)\right)=L_{1}\left(\pi_{\gamma}\right)\right.$ for every $\gamma \in \Gamma_{3}$. Thus we have shown that $R \circ I^{-1} \circ T \circ J$ is an isomorphic embedding of $c_{0}\left(\Gamma_{3}\right)$ into $V_{1}$. This contradicts the fact that $H_{1}$, being separable, satisfies the countable chain condition and the space $C\left(H_{1}\right)$ contains no copy of $c_{0}\left(\Gamma_{3}\right)$ (see $[10$, Thm. 1.4]).

A Banach space $X$ has the separable complementation property if for every separable subspace $X_{1}$ of $X$ there exists a separable and complemented subspace $X_{2}$ which contains $X_{1}$ (see [9]). As a straightforward consequence of the result above we get

COROLlary 8. If a Banach space contains an isomorphic copy of $D(0,1)$, then it does not have the separable complementation property.

Acknowledgments. I wish to thank Professor Lech Drewnowski for stimulating discussions on the subject. 


\section{References}

[1] H. H. Corson, The weak topology of a Banach space, Trans. Amer. Math. Soc. 101 (1961), 1-15.

[2] J. Diestel, Sequences and Series in Banach Spaces, Grad. Texts in Math. 92, Springer, New York, 1984.

[3] L. Drewnowski, Continuity of monotone functions with values in Banach lattices, in: Recent Progress in Functional Analysis, K. D. Bierstedt et al. (eds.), Elsevier, 2001, 185-199.

[4] L. Drewnowski and A. Michalak, Generalized Helly spaces, continuity of monotone functions and metrizing maps, preprint, 2001.

[5] R. Engelking, General Topology, Monografie Mat. 60, PWN-Polish Sci. Publ., Warszawa, 1977.

[6] J. L. Kelley, General Topology, Van Nostrand, Princeton, NJ, 1955.

[7] B. Lavrič, A characterisation of Banach lattices with order continuous norm, Rad. Mat. 8 (1992), 37-41.

[8] W. F. Pfeffer, On some subspaces of the Helly space, Colloq. Math. 35 (1976), 41-46.

[9] A. Plichko and D. Yost, The Radon-Nikodym property does not imply the separable complementation property, J. Funct. Anal. 180 (2001), 481-487.

[10] H. P. Rosenthal, On injective Banach spaces and the spaces $C(S)$, Bull. Amer. Math. Soc. 75 (1969), 824-828.

[11] -, On relatively disjoint families of measures, with some applications to Banach space theory, Studia Math. 37 (1970), 13-36.

[12] P. Wojtaszczyk, Banach Spaces for Analysts, Cambridge Univ. Press, Cambridge, 1991.

Faculty of Mathematics and Computer Science

Adam Mickiewicz University

Umultowska 87

61-614 Poznań, Poland

E-mail: michalak@amu.edu.pl 